\title{
Unveiling Near-Capacity Code Design: The Realization of Shannon's Communication Theory for MIMO Channels
}

\author{
Soon Xin Ng, Jin Wang and Lajos Hanzo \\ School of Electronics and Computer Science, University of Southampton, SO17 1BJ, United Kingdom. \\ Email: $\{$ sxn,lh\}@ecs.soton.ac.uk.
}

\begin{abstract}
In this contribution we show how Shannon's coding theory could be realized for Multiple-Input Multiple-Output (MIMO) channels with the aid of EXtrinsic Information Transfer (EXIT) charts and the Maximum-Aposteriori Probability (MAP) algorithm. We review the relationship between the channel capacity, EXIT charts and the MAP algorithm, outlining the principles of designing near MIMO-channel capacity coding schemes. Both serial and parallel concatenated coding schemes are designed based on these principles and near MIMO-channel capacity performance is achieved.
\end{abstract}

\section{INTRODUCTION}

The history of channel coding dates back to Shannon's pioneering work [1] in 1948, where he showed that it is possible to design a communication system having an indefinitesimally low probability of error, whenever the rate of transmission is lower than the capacity of the channel. This motivated the search quest for codes that would produce arbitrarily small probability of error at a transmission rate close to the channel capacity. The classic coding design aims to approach the channel capacity by optimum channel codes which requires a huge number of trellis states or code memory, using a non-iterative decoder.

A breakthrough in the history of error control coding was the invention of turbo codes by Berrou et al. [2] in 1993. Convolutional codes were used as the component, which were combined with iterative decoders employing the Maximum-Aposteriori Probability (MAP) algorithm [3]-[5]. The results demonstrated that a performance close to the Shannon limit can be achieved in practice with the aid of binary codes using Binary Phase Shift Keying (BPSK) modulation. Turbo codes were later designed also for higher-order modulation. More specifically, bit-based Turbo Coded Modulation (TuCM) [6] and symbol-based Turbo Trellis Coded Modulation (TTCM) [7] were designed for attaining a higher spectral efficiency. The classic design of iterative decoders was based on analysing the associated distance spectrum, which influenced the Bit-Error Ratio (BER) floor of the code [8].

In order to analyze the convergence behaviour of an iterative decoding/detection scheme, density evolution techniques [9] and EXtrinsic Information Transfer (EXIT) charts [10], [11] were proposed. More explicitly, as suggested by the terminology, density evolution tracks the density distribution of the extrinsic probabilities, as the number of decoding iterations increases [12]. Smilarly, EXIT charts track the mutual information exchange between the component decoders in consecutive iterations. It has been shown in [12], [13] that the area between the EXIT curves of the components in an iterative decoder is characteristic of the iterative decoder's ability to approach the channel's capacity. This is often refered to as the 'area-property' of the EXIT charts, which has been used by the binary EXIT chart [10] for rendering a near-capacity binary code design to a curve fitting problem.

The financial support of the EPSRC UK and that of the European Union is gratefully acknowledged.
In this contribution, we present a unified portrayal of the channel capacity and of the symbol-based EXIT charts [11], based on the mutual information formula. We show that the area under the symbolbased EXIT charts is related to the channel capacity. The outline of the paper is as follows. The system model is described in Section II and the EXIT charts is detailed in Section III. Two near-capacity coding schemes are designed in Section IV and our conclusion is offered in Section V.

\section{SySTEM MODEL}

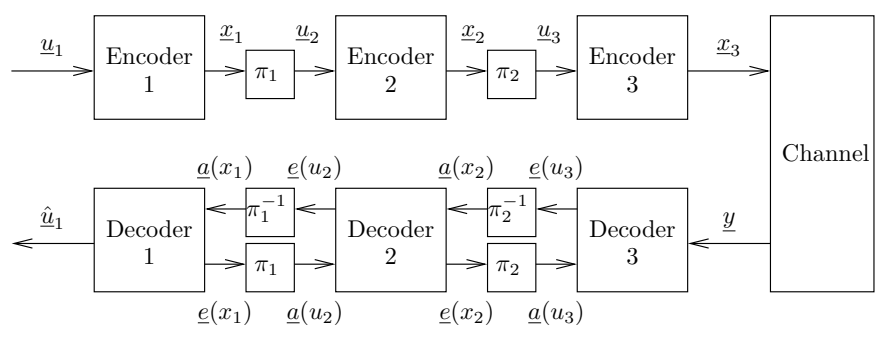

Fig. 1. The block diagram of a 3-stage serially concatenation coding scheme.

Fig. 1 shows a 3 -stage serially concatenated coding scheme, where $\underline{u}_{i}=\left\{u_{i, 1}, u_{i, 2}, \ldots\right\}$ and $\underline{x}_{i}=\left\{x_{i, 1}, x_{i, 2}, \ldots\right\}$ denote the sequences of information symbols and coded symbols of 'Encoder $i$, respectively. The sequence of channel-contaminated received symbols is denoted as $\underline{y}$ and the estimate of $\underline{u}$ is denoted as $\underline{\hat{u}}$. The interleaver and deinterleaver are represented by $\pi$ and $\pi^{-1}$, respectively. The notations $\underline{a}\left(x_{i}\right)=\left\{a\left(x_{i, 1}\right), a\left(x_{i, 2}\right), \ldots\right\}$ and $\underline{e}\left(x_{i}\right)=\left\{e\left(x_{i, 1}\right), e\left(x_{i, 2}\right), \ldots\right\}$ represent the sequences of the a priori and extrinsic probability, respectively, for the coded symbols of 'Decoder $i$ '. Similarly, $\underline{a}\left(u_{i}\right)$ and $\underline{e}\left(u_{i}\right)$ denote the corresponding sequences of a priori and extrinsic probability of the information symbols of 'Decoder $i$ '.

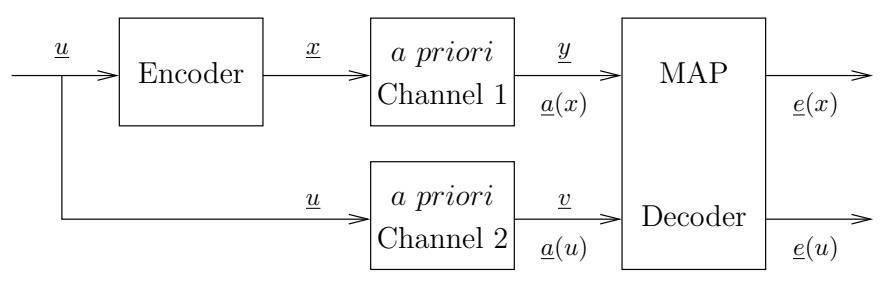

Fig. 2. The encoding and decoding of an intermediate encoder whose input symbols, $\underline{u}$, emenate from an outer encoder and whose output symbols, $\underline{x}$, are fed to an inner encoder. The a priori channels are used to model the outer and inner decoders.

Fig. 2 illustrates the decoding model of the intermediate decoder, i.e. the 'Decoder 2' of Fig. 1. We may use two a priori channels to model the outer and inner decoders following the approach of [12, Fig. 1]. More explicitly, the top a priori channel in Fig. 2 is used to model an inner decoder which has a transition probability of $P\left(y_{k} \mid x_{k}\right)$ signifying the probability of producing $y_{k}$ at its output when the input is $x_{k}$. Similarly, the a priori channel at the bottom 
of Fig. 2 is used to model an outer decoder, which has a transition probability of $P\left(v_{k} \mid u_{k}\right)$, representing the probability of producing $v_{k}$ at its output, when the input is $u_{k}$.

Let us consider a Multiple-Input Multiple-Output (MIMO) system, which invokes $N_{t}$ transmit antennas and $N_{r}$ receive antennas. When complex-valued $\mathcal{M}$-ary PSK/QAM is employed, the received signal vector of the MIMO system can be written as:

$$
\mathbf{y}=\mathbf{H} \mathbf{x}+\mathbf{n}
$$

where $\mathbf{y}=\left[y_{1}, \ldots, y_{N_{r}}\right]^{T}$ is an $N_{r}$-element vector of the received signals, $\mathbf{H}$ is an $\left(N_{r} \times N_{t}\right)$-element channel matrix, $\mathbf{x}=$ $\left[x_{1}, \ldots, x_{N_{t}}\right]^{T}$ is an $N_{t}$-element vector of the transmitted signals and $\mathbf{n}=\left[n_{1}, \ldots, n_{N_{r}}\right]^{T}$ is an $N_{r}$-element noise vector. Each elements of $\mathbf{n}$ is an Additive White Gaussian Noise (AWGN) process having a zero mean and a variance of $N_{0} / 2$ per dimension. There are $M=$ $\mathcal{M}^{N_{t}}$ number of possible $\mathcal{M}$-ary PSK/QAM phasor combinations in the transmitted signal vector $\mathbf{x}$. Let us denote $\mathbf{x}$ as an $M$-ary PSK/QAM signal vector.

The conditional probability of receiving a signal vector $\mathbf{y}$, given that an $M$-ary PSK/QAM signal vector $\mathbf{x}^{(m)}, m \in\{1, \ldots, M\}$, was transmitted over Rayleigh fading channels is determined by the Probability Density Function (PDF) of the noise, yielding:

$$
p\left(\mathbf{y} \mid \mathbf{x}^{(m)}\right)=\frac{1}{\left(\pi N_{0}\right)^{N_{r}}} \exp \left(\frac{-\left\|\mathbf{y}-\mathbf{H} \mathbf{x}^{(m)}\right\|^{2}}{N_{0}}\right)
$$

where $\|()$.$\| is the Frobenius norm of vector (.).$

The capacity of Discrete-input Continuous-output Memoryless Channel (DCMC) [14] for the MIMO system using $M$-ary signalling can be derived as [15], [16]:

$$
C=\log _{2}(M)-\frac{1}{M} \sum_{m=1}^{M} \mathrm{E}\left[\log _{2} \sum_{n=1}^{M} \exp \left(\Psi_{m, n}\right) \mid \mathbf{x}^{(m)}\right],
$$

where the exponent $\Psi_{m, n}$ is given by [16]:

$$
\Psi_{m, n}=\frac{-\left\|\mathbf{H}\left(\mathbf{x}^{(m)}-\mathbf{x}^{(n)}\right)+\mathbf{n}\right\|^{2}+\|\mathbf{n}\|^{2}}{N_{0}} .
$$

\section{EXIT CHARTS}

The EXIT charts [10], [11] visualize the input/output characteristics of the constituent MAP decoders in terms of the average mutual information transfer. Let us use the term 'mutual information' as the 'per-symbol mutual information', unless otherwise stated. In the context of the intermediate decoder of Fig. 2, the EXIT chart visualises the following mutual information exchange:

1) average mutual information of $\underline{u}$ and $\underline{a}(u)$ :

$$
I_{A}(u)=\frac{1}{N_{u}} \sum_{k=1}^{N_{u}} I\left(u_{k} ; a\left(u_{k}\right)\right) ;
$$

2) average mutual information of $\underline{x}$ and $\underline{a}(x)$ :

$$
I_{A}(x)=\frac{1}{N_{x}} \sum_{k=1}^{N_{x}} I\left(x_{k} ; a\left(x_{k}\right)\right)
$$

3) average mutual information of $\underline{u}$ and $\underline{e}(u)$ :

$$
I_{E}(u)=\frac{1}{N_{u}} \sum_{k=1}^{N_{u}} I\left(u_{k} ; e\left(u_{k}\right)\right) ;
$$

4) average mutual information of $\underline{x}$ and $\underline{e}(x)$ :

$$
I_{E}(x)=\frac{1}{N_{x}} \sum_{k=1}^{N_{x}} I\left(x_{k} ; e\left(x_{k}\right)\right)
$$

where the number of symbols in the sequences $\underline{u}$ and $\underline{x}$ are given by $N_{u}$ and $N_{x}$, respectively. Since the intermediate decoder is associated with four mutual information transfers according to Eqs. (5)-(8), two three-dimensional EXIT charts [17], [18] are required for visualising the four-dimensional mutual information transfer between the intermediate decoder (two-input, two-output) and the outer decoder (oneinput, two-output) as well as between the intermediate decoder and the inner decoder (two-input, one output) of Fig. 1.

Provided that a MAP decoder is used, the average extrinsic mutual information of $\underline{u}$ may be computed as [11]:

$$
\begin{aligned}
I_{E}(u) & =\frac{1}{N_{u}} \sum_{k=1}^{N_{u}} H\left(u_{k}\right)-H\left(u_{k} \mid e\left(u_{k}\right)\right) \\
& =\log _{2}\left(\mathcal{M}_{u}\right)-\frac{1}{N_{u}} \sum_{k=1}^{N_{u}} \mathrm{E}\left[\sum_{m=1}^{\mathcal{M}_{u}} e\left(u_{k}^{(m)}\right) \log _{2}\left(e\left(u_{k}^{(m)}\right)\right)\right]
\end{aligned}
$$

where $e\left(u_{k}^{(m)}\right)=P\left(u_{k}^{(m)} \mid \underline{y}, \underline{v}_{[k]}\right)$ is the extrinsic probability of the hypothetically transmitted symbol $u_{k}^{(m)}$, for $m \in\left\{1, \ldots, \mathcal{M}_{u}\right\}$, which is provided by the MAP decoder and the expectation may be removed, when $N_{u}$ is sufficiently large, yielding:

$$
I_{E}(u)=\log _{2}\left(\mathcal{M}_{u}\right)-\frac{1}{N_{u}} \sum_{k=1}^{N_{u}} \sum_{m=1}^{\mathcal{M}_{u}} e\left(u_{k}^{(m)}\right) \log _{2}\left(e\left(u_{k}^{(m)}\right)\right) .
$$

Similarly, we have [11]:

$$
I_{E}(x)=\log _{2}\left(\mathcal{M}_{x}\right)-\frac{1}{N_{x}} \sum_{k=1}^{N_{x}} \sum_{m=1}^{\mathcal{M}_{x}} e\left(x_{k}^{(m)}\right) \log _{2}\left(e\left(x_{k}^{(m)}\right)\right),
$$

where $e\left(x_{k}^{(m)}\right)=P\left(x_{k}^{(m)} \mid \underline{y}_{[k]}, \underline{v}\right)$ is the extrinsic probability of the hypothetically transmitted symbol $x_{k}^{(m)}$, for $m \in\left\{1, \ldots, \mathcal{M}_{u}\right\}$, generated by the MAP decoder and $N_{x}$ is assumed to be sufficiently large.

The average a priori mutual information of $\underline{u}$ and $\underline{x}$ may be model using the following assumptions [11], [19]:

1) the LLRs of the bits are Gaussian distributed: the LLR of a bit $b$, which can be either from the sequence $\underline{u}$ or $\underline{x}$, is given by $[10]$ :

$$
z=h_{A} b+n_{A}
$$

where the variance of the AWGN $n_{A}$ is $\sigma_{A}^{2}$ per dimension and the equivalent 'fading factor' is given by $h_{A}=\sigma_{A}^{2} / 2$ [10];

2) the bits in a symbol are assumed to be independent of each other and uniformly distributed: the average a priori mutual information of a symbol sequence $\underline{u}$ (or $\underline{x}$ ), where each symbol $u_{k}$ (or $x_{k}$ ) consists of $L_{u}$ (or $L_{x}$ ) bits, is $L_{u}$ (or $L_{x}$ ) times the average a priori mutual information of a bit in the symbol.

The average a priori mutual information of a certain bit denoted as $b \in\left\{b^{(1)}=+1, b^{(2)}=-1\right\}$ and its LLR $z$ may be expressed similar to Eq. (3) as:

$$
I(b ; z)=1-\frac{1}{2} \sum_{m=1}^{2} \mathrm{E}\left[\log _{2} \sum_{n=1}^{2} \exp \left(\Psi_{m, n}^{A}\right) \mid b^{(m)}\right],
$$

where $\exp \left(\Psi_{m, n}^{A}\right)=p\left(z \mid b^{(n)}\right) / p\left(z \mid b^{(m)}\right)$ and the conditional Gaussian PDF is given by:

$$
p(z \mid b)=\frac{1}{\sqrt{2 \pi \sigma_{A}^{2}}} \exp \left(\frac{\left(z-h_{A} b\right)^{2}}{2 \sigma_{A}^{2}}\right),
$$

while the exponent is given by:

$$
\Psi_{m, n}^{A}=\frac{-\left|\left(\sigma_{A}^{2} / 2\right)\left(b^{(m)}-b^{(n)}\right)+n_{A}\right|^{2}+\left|n_{A}\right|^{2}}{2 \sigma_{A}^{2}} .
$$


Note that another interpretation of Eq. (12) was given in [10, Eq. (14)]. We have a function $I_{A}=I(b ; z)=J\left(\sigma_{A}\right)$, with $J\left(\sigma_{A}\right)$ being monotonically increasing and therefore invertible. Hence, at a given $I_{A}$ we may find the corresponding $\sigma_{A}$ value from $J^{-1}\left(I_{A}\right)$. Finally one may compute the corresponding LLR value $z$ from Eq. (11). The a priori mutual information of a $L_{u}$-bit symbol $u_{k}$ is given by:

$$
I\left(u_{k} ; z_{(k)}\right)=\sum_{i=1}^{L_{u}} I\left(b_{(k, i)}^{u} ; z_{(k, i)}^{u}\right),
$$

where $z_{(k)}^{u}=\left\{z_{(k, 1)}^{u}, \ldots, z_{\left(k, L_{u}\right)}^{u}\right\}$ is the LLR sequence, which is related to the $L_{u}$ bits of $u_{k}$ and $z_{(k, l)}^{u}$ is the LLR of $b_{(k, l)}^{u}$, which is the $l$ th bit in the $k$ th symbol $u_{k}$.

\section{NEAR-CAPACITY CODE DESIGN}

It is clear from Eqs. (3), (9), (10) and (12) that both the computation of the channel capacity and that of the EXIT chart is based on the the same mutual information formula. It was shown in [11], [12] that when a MAP decoder is used, the extrinsic probability $e\left(u_{k}\right)$, which is computed at the decoder's output, contains the same amount of information as the sequences $\underline{y}$ and $\underline{v}_{[k]}$ at the decoder's input, i.e. we have:

$$
I\left(u_{k} ; e\left(u_{k}\right)\right)=I\left(u_{k} ; \underline{y}, \underline{v}_{[k]}\right) .
$$

Similarly, the extrinsic probability $e\left(x_{k}\right)$ contains the same amount of information as the sequences $\underline{y}_{[k]}$ and $\underline{v}$ :

$$
I\left(x_{k} ; e\left(x_{k}\right)\right)=I\left(x_{k} ; \underline{y}_{[k]}, \underline{v}\right) .
$$

Hence, the area under the EXIT curve $I_{A}$ is the same as the area under the EXIT curve $I_{E}$ when the MAP algorithm is used in all decoders, although the shape of the curve may change. In other words, the MAP decoder acts like a lossless filter where its two outputs represent a full statistical characterisation of the two sequences observed at its input.

According to the properties of EXIT charts [12], the area under the EXIT curve of the inner code equals to the capacity of the communication channel (the upper a priori channel in Fig. 2), when the communication channel's input is given by equiprobable $M$-ary symbols and the a priori channel (the lower a priori channel in Fig. 2) is modeled using a Binary Erasure Channel (BEC) [12]. This area property was formally shown to be valid for arbitrary inner codes and communication channels, provided that the a priori channel is modeled by a BEC [12]. Furthermore, the area under the EXIT curve of the outer code equals to $\left(1-R_{1}\right)$, where $R_{1}$ is the outer code's rate.

Let us consider a PSK/QAM MIMO mapper as the inner encoder ('Encoder 3' of Fig. 1) and assume that 'Decoder 3' is a MIMO softdemapper. The decoder can be modelled using Fig. 2 where the upper a priori channel is an uncorrelated Rayleigh fading MIMO channel and the lower a priori channel models 'Decoder 2' of Fig. 1. Since the MIMO channel is non-dispersive and uncorrelated in time, the a posteriori probability of the soft MIMO demodulator contains only the channel transition term $\gamma_{k}(\grave{s}, s)$ of the MAP algorithm:

$$
o\left(u_{k}\right)=\gamma_{k}(\grave{s}, s)=a\left(x_{k}\right) \cdot a\left(u_{k}\right) \text {. }
$$

Hence, the associated symbol-based extrinsic probability is given by $e\left(u_{k}\right)=o\left(u_{k}\right) / a\left(u_{k}\right)=a\left(x_{k}\right)$, which is independent of the sequence $\underline{v}$. Hence, based on Eq. (16) we have:

$$
I\left(u_{k} ; e\left(u_{k}\right)\right)=I\left(u_{k} ; y_{k}\right) .
$$

In this case, $I_{E}(u)$ is independent of $I_{A}(u)$, where $I_{E}(u)$ is a constant across $I_{A}(u)=\left\{0, \ldots, I_{A, \max }\right\}$, with $I_{A \text {, max }}$ being the capacity of the lower a priori channel. Since 'Encoder 3' represents a one-to-one mapper, we have $I\left(u_{k} ; y_{k}\right)=I\left(x_{k} ; y_{k}\right)$. Hence, the area under the EXIT curve of this soft demodulator can be computed using Eqs. (8) and (19) as:

$$
\begin{aligned}
\mathcal{A} & =\int_{0}^{I_{A, \max }} \frac{1}{N} \sum_{k=1}^{N} I\left(u_{k} ; e\left(u_{k}\right)\right) d I_{A} \\
& =\int_{0}^{I_{A, \max }} d I_{A} \frac{1}{N} \sum_{k=1}^{N} I\left(x_{k} ; y_{k}\right) \\
& =I_{A, \max } I(x ; y)=I_{A, \max } C,
\end{aligned}
$$

where $C$ is the capacity of the MIMO channel (including the case when $N_{t}=N_{r}=1$ ) given by Eq. (3). Hence, it is shown that the area under the symbol-based EXIT curve of a soft-demapper is given by the product of the communication channel's capacity $C$ and the capacity of the a priori channel $I_{A \text {,max }}$. This area property derived for the related symbol-based EXIT chart also indicates that when the intermediate code (or the outer code of a 2-stage scheme) is also symbol-based, all the mutual information gleaned from the communication channel has been transfered to the soft-demapper's extrinsic symbol probability sequence. In this case, the link from $\underline{x}_{2}$ of 'Encoder 2' to $\underline{a}\left(x_{2}\right)$ of 'Decoder 2' in Fig. 1 may be considered to be a transformed MIMO channel, namely $I_{A}\left(x_{2}\right)=I\left(x_{2} ; a\left(x_{2}\right)\right)=$ $C$. Hence, if we employ a symbol-based 'Encoder 2' and a MAP algorithm for all decoders, then the area under the EXIT curve of $e\left(u_{2}\right)$ at the output of 'Decoder 2' can be shown to be:

$$
\mathcal{A}=C \cdot I_{A}\left(u_{2}\right) \text {. }
$$

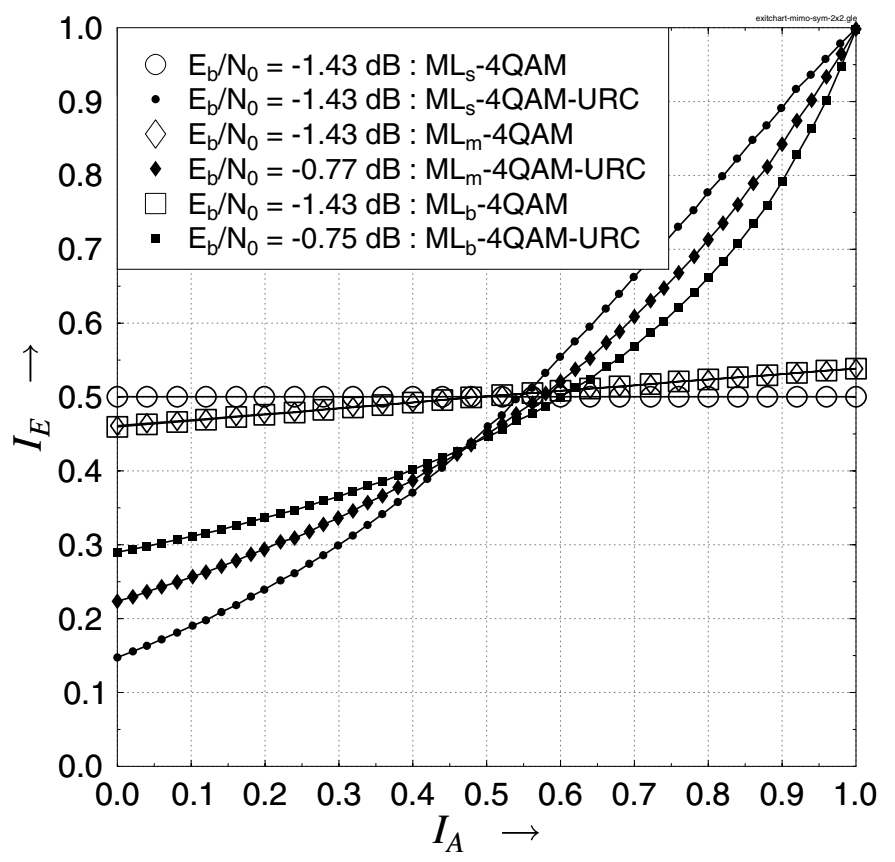

Fig. 3. Normalized EXIT charts where the area under each EXIT curve is 0.5 . The different soft-demapper types that compute the extrinsic probability of 4-bit, 2-bit or 1-bit symbols are represented as $\mathrm{ML}_{s}, \mathrm{ML}_{m}$ and $\mathrm{ML}_{b}$, respectively.

Let us now consider a $N_{t}=N_{r}=2$ MIMO scheme using $\mathcal{M}=$ 4-level QAM transmissions. The MIMO channel's capacity computed from Eq. (3) at a throughput of $\eta=2 \mathrm{bit} / \mathrm{s} / \mathrm{Hz}$ is $E_{b} / N_{0}=-1.43 \mathrm{~dB}$. The MIMO mapper is considered to be the inner encoder (Encoder 3 of Fig. 1), where $L=N_{t} \times \log _{2}(\mathcal{M})=4$ bits are mapped to $N_{t}=2$ transmit antennas, each employing 4QAM transmissions. 
Note that we may represent the input symbol of the MIMO mapper as $N$ number of $L=4$-bit symbols, $2 N$ number of $L / 2=2$-bit symbols or $4 N$ number of $L / 4=1$-bit symbols. At the receiver, the soft-demapper is used to compute the extrinsic probability of these 4bit, 2-bit or 1-bit symbols. The EXIT curves recorded for the various soft-demapper types are shown in Fig. 3 which were normalized to unity by dividing the $I_{A}$ ( or $I_{E}$ ) values by $I_{A, \max }=L=4$ bits. The different soft-demapper types, which compute the extrinsic symbol probability as that of 4-bit, 2-bit or 1-bit symbols are represented as $\mathrm{ML}_{s}, \mathrm{ML}_{m}$ and $\mathrm{ML}_{b}$, respectively. The area under each of the EXIT curves in Fig. 3 equals 0.5 (or $\mathcal{A}=0.5 \times L^{2}=2 L$ before the normalization). It is shown in Fig. 3 that the EXIT curve for $\mathrm{ML}_{s}-4 \mathrm{QAM}$ is a horizontal line at $I_{E}=0.5$, while that for $\mathrm{ML}_{m^{-}}$ 4QAM and $\mathrm{ML}_{b}-4 \mathrm{QAM}$ emerges from $I_{E}<0.5$ and terminates at $I_{E}>0.5$.

\section{A. Serial Concatenated Scheme}

We employed a recursive symbol-based unit-memory Unity-Rate Code (URC) [18] as the intermediate encoder, where each input/output symbol has $L=N_{t} \times \log _{2}(\mathcal{M})=4$ bits and each URC encoded symbol is mapped to $N_{t}=2$ transmit antennas, where 4QAM transmission is used at each antenna. The symbol-based URC encoder employs a modulo- $M=2^{L}$ adder and it requires $M$ number of trellis states. At the receiver a soft-demapper was used to compute the $M=2^{L}=16$-valued extrinsic symbol probability for each of the URC encoded symbol $x_{2, k}$ for $k \in\{1, \ldots, N\}$, where $N=N_{x}=N_{u}$ is the number of symbols. These symbol probabilities are fed to the URC decoder in order to compute the extrinsic probability of the URC's input information symbols $u_{2, k}$ for $k \in\{1, \ldots, N\}$. The EXIT curve of the $\mathrm{ML}_{s}$-4QAM-URC scheme is shown in Fig. 3, which is based on the URC's input information symbol $u_{2}$ while assumming the absence of iterative information exchange with the soft-demapper. Observe in Fig. 3 that the $\mathrm{ML}_{s^{-}}$ 4QAM-URC scheme requires the same $E_{b} / N_{0}$ value as the $\mathrm{ML}_{s}-$ 4QAM arrangement in order to maintain an area of 0.5 under it.

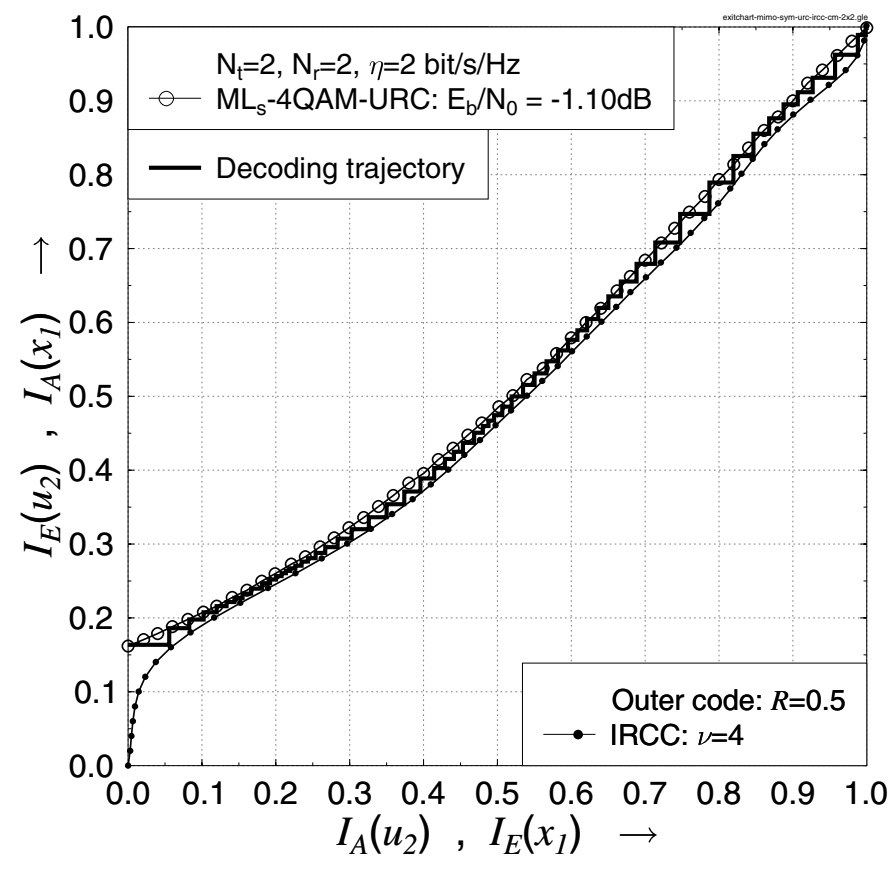

Fig. 4. Normalized EXIT charts for $\mathrm{ML}_{s}-4 \mathrm{QAM}-U R C$ and IRCC.

The EXIT curves of the $\mathrm{ML}_{m}-4 \mathrm{QAM}-\mathrm{URC}$ and $\mathrm{ML}_{b}-4 \mathrm{QAM}-\mathrm{URC}$ schemes are also shown in Fig. 3, where the number of bits per symbol entered into the URC encoder is given by $L / 2=2$ and $L / 4=1$, when employing the $\mathrm{ML}_{m}$ and $\mathrm{ML}_{b}$ scheme, respectively. When there is no iteration between the soft-demapper and the URC decoder, the EXIT curve of the URC decoder depends on the first $I_{E}$ value of the soft-demapper. Hence, both the $\mathrm{ML}_{m}-4 \mathrm{QAM}-\mathrm{URC}$ and $\mathrm{ML}_{b}$-4QAM-URC schemes require a higher $E_{b} / N_{0}$ value in order to maintain an area of 0.5 under their EXIT curves, as shown in Fig. 3. In other words, this loss of information (or $E b / N_{0}$ value) cannot be recovered if there is no iteration between the URC decoder and the soft-demapper. In this case, we only need a 2D EXIT chart in order to analyze the decoding convergence between the $\mathrm{ML}_{s}-4 \mathrm{QAM}-\mathrm{URC}$ scheme and the outer code.

We employ the IRregular Convolutional Codes (IRCCs) of [20] as the outer encoder, where 17 subcodes were constructed from a memory-four mother code. We design the IRCC to match its EXIT curve to that of the $\mathrm{ML}_{s}-4 \mathrm{QAM}-\mathrm{URC}$ scheme fed with the information symbols $u_{2, k}$ for $k \in\{1, \ldots, N\}$. The resultant normalized EXIT curves are shown in Fig. 4, where an EXIT tunnel leading to the maximum $I_{E}\left(x_{1}\right)$ value was created for $E_{b} / N_{0}$ values in excess of $-1.10 \mathrm{~dB}$, which is as close as within $0.33 \mathrm{~dB}$ from the MIMO channel's capacity. The corresponding IRCC weighting coefficients are given by: $\alpha_{o}=\left[0.172469 z^{3}+0.252871 z^{5}+\right.$ $\left.0.214169 z^{9}+0.209495 z^{13}+0.15104 z^{17}\right]$, where the superscript $s=\{3,5,9,13,17\}$ of $z^{s}$ denotes the index of the subcodes used.

\section{B. Parallel Concatenation Scheme}

In contrast to the serial concatenated scheme of the previous section, here we assume that 'Encoder 1' and 'Encoder 2' are parallel concatenated as an outer code, which is the classic Turbo Code (TC) arrangement. The 'Encoder 3' is the same MIMO mapper that was used in Fig. 3. As argued earlier, it is impossible to recover the information loss, when a bit-based outer encoder (or when a bitbased interleaver) is used unless there are iterations between the outer decoder and the inner decoder (soft-demapper), when communicating over MIMO channels. However, a symbol-based TC that exhibits a horizontal EXIT curve, which matches the $\mathrm{ML}_{s}-4 \mathrm{QAM}$ EXIT curve would require non-identical and symbol-based component codes in the TC.

Let us consider a scheme where apart from having inner iterations in the outer TC decoder, there are also outer iterations between the TC decoder and the MIMO soft-demapper. We consider having identical memory $\nu=3$ component codes for the outer TC, where the component code's generator polynomial is given by $G=\left[\begin{array}{ll}13 & 6\end{array}\right]_{8}$, which has a similar structure to the TTCM scheme of [7] except that a bit-interleaver is used before the MIMO mapper ('Encoder 3'). The normalized EXIT curves of the $\mathrm{ML}_{b}-4 \mathrm{QAM}$ and TC decoder (which uses 16 TC iterations) is shown in Fig. 5, where the notation $x_{1 \& 2}$ is used to denote the TC-encoded symbol. An open EXIT tunnel leading to the maximum value of $I_{E}\left(x_{1 \& 2}\right)=1$ was created at $E_{b} / N_{0}=-0.85 \mathrm{~dB}$, which is only $0.58 \mathrm{~dB}$ away from the MIMO channel's capacity.

The normalized EXIT curves of the optimal maximal-distance Non-Systematic Convolutional Codes (NSCCs) [21] having a code polynomial of $G=\left[\begin{array}{ll}15 & 17\end{array}\right]_{8}$ (code memory $\nu=3$ ) and $G=$ [10533 17661$]_{8}$ (code memory $\nu=12$ ) are also shown in Fig. 5. Note that approximately seven outer iterations are required between the TC decoder and the $\mathrm{ML}_{b}-4 \mathrm{QAM}$ for attaining $I_{E} \approx 1$. Each TC component code has $2^{\nu}=8$ trellis states, hence after 16 TC iterations and 7 outer iterations, the $\mathrm{ML}_{b}-4 \mathrm{QAM}-\mathrm{TC}$ scheme will have traversed through $2 \times 2^{\nu} \times 16 \times 7=1792$ number of trellis states per decoded bit. By contrast, the memory-12 maximal-distance NSCC decoder 


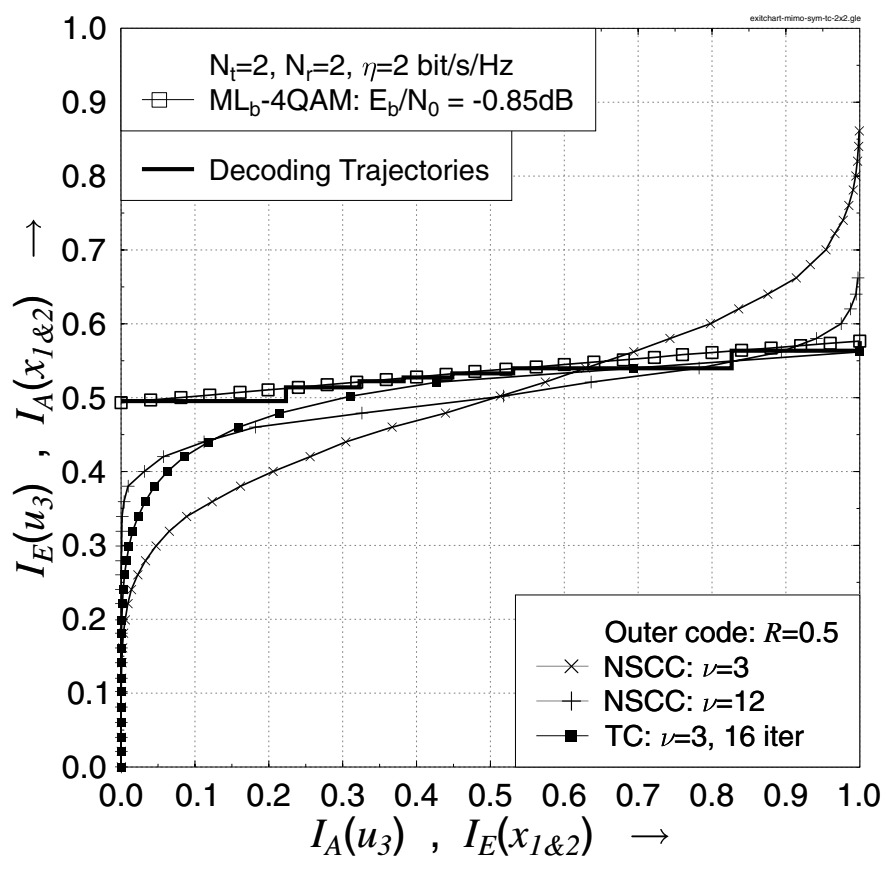

Fig. 5. Normalized EXIT charts for $\mathrm{ML}_{b}-4 \mathrm{QAM}, \mathrm{TC}$ and NSC.

evaluated $2^{12}=4096$ number of trellis states per decoded bit. Despite its higher complexity, there was no open EXIT tunnel leading to $I_{E}\left(x_{1 \& 2}\right)=1$ between the EXIT curves of either of the two NSCC decoders and the $\mathrm{ML}_{b}-4 \mathrm{QAM}$ scheme at $E_{b} / N_{0}=-0.85 \mathrm{~dB}$. Hence, the memory-12 optimal NSCC requires about 4096/1792 $\approx 2.3$ times higher complexity than the $\mathrm{ML}_{b}-4 \mathrm{QAM}-\mathrm{TC}$ scheme, and yet fails to achieve decoding convergence to infinitesimally low BER at $E_{b} / N_{0}=-0.85 \mathrm{~dB}$.

\section{CONCLUSIONS}

We have shown the relationship between the computation of the channel capacity, EXIT charts and the MAP algorithm. As seen in Fig. 4, the classic optimal coding design (i.e. NSCC) was inefficient as it is very difficult to achieve a near-horizontal EXIT curve for matching the EXIT curve of the soft-demapper. It was shown in Fig. 3 that when communicating over MIMO channels, we need outer iterations exchanging extrinsic information with the soft-demapper unless a symbol-based decoder is employed, if approaching the MIMO channel's capacity is desired. It was shown that Shannon's communication theory could be realized for MIMO channels with the aid of the MAP algorithm as well as by using an EXIT charts aided code design and iterative decoding between two or three sub-optimal codes. Hence, achieving near-capacity performance is feasible, when the associated encoding/decoding complexity and decoding delay is affortable. The new challenge for code design is to approach the attainable rate at the lowest encoding/decoding complexity and the lowest possible interleaving delay.

\section{REFERENCES}

[1] C. E. Shannon, "A mathematical theory of communication," Bell System Technical Journal, pp. 379-427, 1948.

[2] C. Berrou and A. Glavieux and P. Thitimajshima, "Near Shannon limit error-correcting coding and decoding: Turbo codes," in Proceedings of the International Conference on Communications, (Geneva, Switzerland), pp. 1064-1070, May 1993.

[3] L.R. Bahl and J. Cocke and F. Jelinek and J. Raviv, "Optimal decoding of linear codes for minimising symbol error rate," IEEE Transactions on Information Theory, vol. 20, pp. 284-287, March 1974.
[4] S. Benedetto, D. Divsalar, G. Motorsi, and F. Pollara, "A soft-input softoutput app module for iterative decoding of concatenated codes," IEEE Communication Letters, pp. 22-24, 1997.

[5] L. Hanzo, T.H. Liew and B.L. Yeap, Turbo Coding, Turbo Equalisation and Space Time Coding for Transmission over Wireless channels. New York, USA: John Wiley IEEE Press, 2002.

[6] S. L. Goff, A. Glavieux, and C. Berrou, "Turbo-codes and high spectral efficiency modulation," in Proceedings of IEEE International Conference on Communications, pp. 645-649, 1994.

[7] P. Robertson and T. Wörz, "Bandwidth-efficient turbo trellis-coded modulation using punctured component codes," IEEE Journal on Selected Areas in Communications, vol. 16, pp. 206-218, Feb 1998.

[8] L. Perez, J. Seghers, and D. Costello, "A distance spectrum interpretation of turbo codes," IEEE Transactions on Information Theory, vol. 42, pp. 1698-1709, November 1996.

[9] R. Gallager, "Low-density parity-check codes," IEEE Transactions on Information Theory, pp. 21-28, 1962.

[10] S. ten Brink, "Convergence behaviour of iteratively decoded parallel concatenated codes," IEEE Transactions on Communications, vol. 49, pp. 1727-1737, October 2001.

[11] J. Kliewer, S. X. Ng, and L. Hanzo, "Efficient computation of exit functions for non-binary iterative decoding," IEEE Transactions on Communications, vol. 54, pp. 2133-2136, December 2006.

[12] A. Ashikhmin, G. Kramer, and S. ten Brink, "Extrinsic information transfer functions: model and erasure channel properties," IEEE Transactions on Information Theory, vol. 50, pp. 2657- 2673, November 2004.

[13] S. ten Brink, "Rate one-half code for approaching the Shannon limit by 0.1 dB," IEE Electronics Letters, vol. 36, pp. 1293-1294, July 2000.

[14] J. G. Proakis, Digital Communications. New York: Mc-Graw Hill International Editions, 3rd ed., 1995.

[15] T. M. Cover and J. A. Thomas, Elements of Information Theory. New York, USA: John Wiley IEEE Press, 1991.

[16] S. X. Ng and Hanzo, "On the mimo channel capacity of multidimensional signal sets," IEEE Transactions on Vehicular Technology, vol. 55, pp. 528-536, March 2006.

[17] M. Tüchler, "Convergence prediction for iterative decoding of threefold concatenated systems," in GLOBECOM '02, (Taipei, Taiwan), pp. 13581362, 17-21 November 2002.

[18] S. X. Ng, J. Wang, M. Tao, L.-L. Yang and L. Hanzo, "Iteratively decoded variable-length space-time coded modulation: code construction and convergence analysis," IEEE Transactions on Wireless Communications, vol. 6, pp. 1953-1963, May 2007.

[19] I. Land, P. Hoeher, and S. Gligorević, "Computation of symbol-wise mutual information in transmission systems with $\log \mathrm{APP}$ decoders and application to EXIT charts," in Proc. International ITG Conference on Source and Channel Coding (SCC), (Erlangen, Germany), pp. 195-202, Jan. 2004.

[20] M. Tüchler, "Design of serially concatenated systems depending on the block length," IEEE Transactions on Communications, vol. 52, pp. 209218, February 2004.

[21] S. Lin and D. Constello Jr., Error Control Coding: Fundamentals and Applications. Englewood Cliffs, NJ, USA: Prentice-Hall, October 1982. ISBN: 013283796X. 